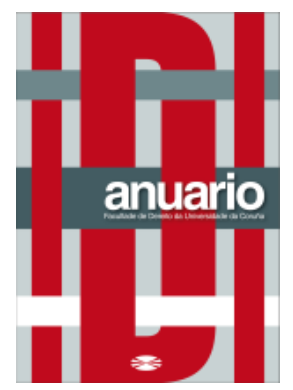

Anuario da Facultade de Dereito da Universidade da Coruña

Vol. 22 (2018), pp. 475-479

ISSNe: 2530-6324 || ISSN: 1138-039X

DOI: https://doi.org/10.17979/afdudc.2018.22.0.5199

\title{
JAVIER RUIPÉREZ, El Título $X$ de la Constitución Española de 1978 a la luz de las funciones de la Reforma Constitucional (Un estudio de Teoría del Derecho Constitucional en cuanto que ciencia conceptual y ciencia práctica), VLEX, México, 2017.
}

\author{
ENRIQUE SÉPTIMO RODRÍGUEZ \\ Profesor de la Facultad de Derecho de la Universidad Autónoma del Estado de México
}

En palabras de Javier Ruipérez, su obra antes citada constituye una monografía que persigue como fin rendir un primer y urgente homenaje a su Maestro y mentor, Pedro De Vega, estudioso de la Teoría del Estado y del Derecho Constitucional, quien sostenía que a través de éste, se pueden hacer efectivas las viejas ideas de "Democracia", "Libertad" e "Igualdad". La obra se desprende de una invitación que Víctor Alejandro Wong Meraz, le realizó a Ruipérez, para incluir una aportación en la obra colectiva "La problemática de la 
Reforma Constitucional: Análisis de procesos de reforma en América y Europa"; la aportación se encontraba lista y a punto de ser remitida a la editorial, cuando en fecha veintisiete de abril del año dos mil dieciséis, sobrevino el fallecimiento del Doctor Pedro De Vega, motivo por el cual Alejandro Wong sabedor de la estrecha relación académica y personal que lo unía con Javier Ruipérez, lo exhortó a que la contribución que se tenía contemplada para la obra colectiva se publicara a manera de un justo homenaje en una obra independiente, propuesta que fue aceptada.

Como se ha señalado, la obra de Javier Ruipérez que en este texto se reseña, constituye un homenaje al Profesor Pedro De Vega, quien trascendió en el campo del Derecho Constitucional Iberoamericano como teórico de las Ciencias del Estado y Ciencias del Derecho del Estado, además de que guió su actuar de manera objetiva y cabal como los grandes constitucionalistas científicos, Georg Jellinek o Hermann Heller, hacia una dimensión teórico práctica. En este sentido, dentro de la obra que se analiza Javier Ruipérez, discípulo de Pedro De Vega, expone sus enseñanzas de una manera precisa sobre la problemática que se genera en torno a la reforma constitucional, lo cual realiza a lo largo de seis apartados.

El primero se titula "Una previa y necesaria, cuestión metodológica: sobre la Teoría del Derecho Constitucional como contenido de las Ciencias del Estado y de las Ciencias del Derecho del Estado, y su doble dimensión como ciencia jurídica conceptual y práctica", aquí el autor parte de la necesidad de abordar las concepciones de Jellinek sobre las Ciencias Constitucionales, por ser uno de los más grandes Maestros de las Ciencias Jurídicas, quien en su Teoría general del Estado, sintetizó de manera excepcional la Teoría del Estado de la última centuria, convirtiéndose esto en un patrimonio científico. Por tal motivo las concepciones vertidas por Jellinek han sido gran influencia para los más brillantes, lucidos y capaces estudiosos de la problemática del Estado, la Política y el Derecho, constituyéndose así en el forjador de la Ciencia del Derecho Constitucional en Europa.

En este sentido, Javier Ruipérez trata de justificar las apelaciones hechas a las concepciones jellinekianas, las cuales señala, han tratado de ser descalificadas por la práctica política de aquellos con actitud antidemócrata, al menos desde tres perspectivas; en primer término con la reprobación de acudir a las construcciones de los clásicos realizadas por aquellos Profesores/Profesoras de Derecho Constitucional, ya que pretender encontrar soluciones a los problemas actuales en las formulaciones de aquellos, es tanto como tratar de hacer magia negra, toda vez que para éstos las únicas fuentes de conocimiento son los diversos pronunciamientos que hayan podido emanar del Tribunal Constitucional. En segundo lugar, para Ruipérez se encuentran aquellos que dada su propia incapacidad para comprender el contenido material de la obra prefieren proceder a la descalificación total de los trabajos 
elaborados por otros constitucionalistas. Y por último se encuentran los profesionales del estudio del Derecho Constitucional, que alguna vez han servido como Letrados del Tribunal Constitucional, mismos que pretenden reducir la investigación científica en el ámbito de la Teoría del Estado y de la Constitución a la mera transpiración de los fundamentos jurídicos de las diversas sentencias del Tribunal Constitucional.

Para concluir este apartado atinadamente el autor, en atención a lo expuesto por Jellinek en su obra "Reforma y mutación de la Constitución", concluye que la auténtica labor del investigación científica en el ámbito del Derecho Constitucional requiere del conocimiento político para obtener un resultado crítico, por lo que la Teoría del Estado, la Ciencia Política, la Sociología, la Filosofía del Estado, la Teoría Política, la Historia e incluso las Ciencias Económicas comprendidas como Ciencias del Estado no pueden ser materias ajenas al jurista. Por el contrario, el Profesor Ruipérez sostiene que el constitucionalista necesita de su conocimiento y su práctica para ejercer adecuadamente su misión.

El autor en un segundo apartado intitulado "Consecuencias de lo anterior par el estudio del Instituto de la Reforma Constitucional en cuanto que contenido de la Teoría del Estado y de la Constitución y de la Teoría del Derecho Constitucional", pone de manifiesto sus conocimientos sobre la construcción científica del Derecho Constitucional, realizando un análisis deductivo que aterriza en el cómo ha satisfecho la solución normativa contenida en el Título X de la Constitución española de 1978 las funciones que ha de cumplir el procedimiento de revisión constitucional en el marco teórico práctico; lo cual remite directamente a las modificaciones formales de la Constitución, Verfassungsänderung, que para Javier Ruipérez este puede ser definido (siguiendo los planteamiento de Carlo Cereti, Paolo Biscaretti di Ruffia, Karl Loewenstein, Pedro De Vega y Rogelia Calzada Conde), como "una operación normativa, voluntaria e inmediata, tendiente a modificar, parcial o totalmente, una Constitución a través de órganos especiales, o procedimientos diversos de los establecidos para la legislación ordinaria”.

En este sentido para el autor, el Verfassungsänderung comprende la reforma que se concreta en una actividad normativa que modifica total o parcialmente el texto de la Constitución, de donde resulta evidente que ésta operación se traducirá en la adición, cambio o supresión de una o varias palabras, frases o párrafos de uno o varios artículos o incluso de la totalidad de los artículos de la Constitución. También el Verfassungsänderung comprende la institución de la revisión constitucional, colocando el autor como el más claro ejemplo a la tan reconocida sentencia del caso "Marbury versus Madison" con la cual el Juez Marshall subsana el olvido del Poder Constituyente, dando así origen al judicial review. Por lo anterior, el Profesor Javier Ruipérez resalta la importancia de que todo jurista, aunque no se trate de un constitucionalista o práctico de la política, deba conocer, la institución de la reforma y revisión constitucional, puesto que no se debe olvidar su 
trascendente función en la vida política y jurídica. Con base en lo anterior, y siguiendo el planteamiento de Don Pedro De Vega, el autor Javier Ruipérez a lo largo de cuatro apartados más desarrolla la problemática de la reforma constitucional, esquematizándolo de la siguiente manera:

- "La técnica de la revisión constitucional como instrumento para la corrección de errores técnicos”. Aquí el autor sostiene que el poder de revisión constitucional (que se encuentra legitimado para actuar en el nivel normativo supremo) puede proceder a la corrección de los errores técnicos en los que pudo incurrir el Legislador Constituyente, en el proceso de elaboración, discusión y aprobación de la Ley Fundamental. Esta figura es estudiada por el autor a la luz del Título $\mathrm{X}$ de la Constitución española, donde presenta gran dinamismo jurídico y político, en virtud de que, a través de esta se puede llevar a cabo la corrección de cuantos errores técnicos pudieran existir en la primigenia norma establecida por el constituyente de 1977-1978.

Mediante este instrumento jurídico, el Texto Constitucional logra obtener una vigencia real, mismo que se habría aprobado en su origen para que fuese eterno, planteamiento que Javier Ruipérez retoma de Kelsen, pero que necesaria e inevitablemente debe ser modificado, en este sentido, la norma Constitucional tiene que ser enjuiciada cada cierto tiempo, pues con el propio devenir histórico se podría advertir de la existencia de algún error técnico que pueda dificultar la exacta observancia de los principios constitucionales.

- "La reforma constitucional como mecanismo para el mantenimiento de la realidad constitucional". A través de esta función que ha de cumplir el instituto del Verfassungsänderung dentro del Estado Constitucional democrático y social, como lo sostiene el autor de la obra, se logra el mantenimiento y adecuación entre la realidad jurídico-normativa (que es la que se plasma en el Texto Constitucional) y la realidad política, social y económica subyacente. Dicha función es la más conocida y a la que más se recurre por parte de los prácticos de la política y por parte de los profesionales científicos del Estado, la Política y el Derecho. El autor sostiene que a través de la reforma se mantiene vigente la Constitución y que solamente de esta única manera "el Código Jurídico-Político Fundamental tan sólo podrá desplegar toda su potencialidad normativa, y, en definitiva, únicamente podrá afirmarse que goza de una vigencia real y efectiva, en la que en la medida en que la Comunidad Política de que se trate se mantenga, en la medida de lo posible, la perfecta adecuación entre el contenido formalizado en el documento de gobierno y la realidad política, social y económica subyacente y que aquel pretende regular...". A través de este pensamiento coherente y concreto de Ruipérez, se entiende la importancia trascendencia de la institución de la reforma constitucional, pues es el mecanismo idóneo para adecuar la Norma Fundamental a la tan cambiante realidad política.

Precisamente en el planteamiento anterior, radica la postura del autor de que esto constituye una de las más importantes dificultades del Derecho Constitucional (cómo conseguir la 
adecuación de la Norma Fundamental a la realidad política, social y económica subyacente), la cual eminentemente según su postura tiene que ser abordada a partir del ámbito teórico pero sobre todo práctico. Como bien lo refiere el autor de la obra, después de estos razonamientos, la reforma constitucional adquiere sentido y un significado muy concreto, que hace incuestionable su utilización en el Derecho Constitucional.

- "La revisión constitucional como técnica de salvaguardia de la voluntad soberana del Poder Constituyente frente a la actuación de los Poderes Constituidos (La conciliación entre el principio democrático y el principio de supremacía constitucional)". En cuanto hace a esta función de la reforma constitucional, el Profesor Ruipérez, brillantemente expone que en atención al principio de legalidad los poderes constituidos del Estado pueden realizar modificaciones al Texto Constitucional que se encuentra en vigor en una determinada realidad social, y que incluso estas modificaciones se pueden encontrar encaminadas al establecimiento de un nuevo régimen político, consecuentemente a un establecimiento de un orden constitucional distinto; es aquí donde el autor atinadamente guiado por el pensamiento del Maestro De Vega, precisa que estos sujetos sólo se encuentran legitimados para el desarrollo de un poder político limitado, situación que no acontece en aquellos Estados donde la tradición constitucionalista es nula o muy precaria. En este orden de ideas la reforma constitucional debe de ser empleada como un mecanismo de defensa de las decisiones fundamentales y de los valores que inspiraron a la Constitución, por lo tanto como lo sostiene el autor de la obra que se reseña, "ningún jurista puede ignorar... que la técnica de la reforma no nace tanto para permitir el cambio, cuanto para tratar de preservar y, de una u otra suerte, perpetuar la voluntad soberana del Poder Constituyente."

Como una posible solución al problema señalado en el párrafo que antecede se instauro la idea del principio de la rigidez constitucional, el cual fue defendido según el autor por Pedro De Vega a lo largo de toda su obra, en atención a este principio se establece un proceso distinto de reforma (más agravado) para las disposiciones constitucionales que para las leyes ordinarias. Javier Ruipérez cita a Georges Vedel de manera atinada, pues este último refiere que la rigidez constitucional se presenta como una consecuencia de la supremacía constitucional, en otras palabras, esta característica es la que diferencia a una Norma Constitucional de una Ordinaria. Todo lo anterior, se traduce en que la soberanía popular convertida en Poder Constituyente queda a salvo cuando se encuentra frente a la voluntad del legislador, respecto a esta idea el autor oportunamente cita a Hamilton quien en su obra intitulada el "El Federalista" refiere: "Y si ocurriese que entre las dos hay un discrepancia, debe preferirse, como es natural, aquella que posee fuerza obligatoria y validez superior; en otras palabras, debe preferirse la Constitución a la ley ordinaria, la intención del pueblo a la intención de sus mandatarios". Con la idea anterior, el Profesor Ruipérez deja en claro que el actuar de los poderes constituidos tiene límites, siendo el principal de estos la voluntad del soberano. 
- "La técnica de la reforma constitucional como instrumento para la articulación de la continuidad jurídica del Estado. La problemática de los límites materiales a la actuación del poder de revisión, y los controles jurídicos a la reforma de la constitución". Como lo ha sostenido Javier Ruipérez a lo largo de su tan brillante obra que en este breve texto se reseña, la Verfassungsänderung es un mecanismo a través del cual la realidad jurídica se adapta a la realidad política, pero sobretodo se constituye en un mecanismo idóneo para lograr la articulación de la continuidad jurídica del Estado, toda vez que como se ha señalado anteriormente la revisión constitucional es el instrumento idóneo para atender la dinamicidad política que el devenir del tiempo impone a la norma fundamental; pero esta figura debe de actuar según lo expuesto por el autor bajo ciertos límites expresos o en otras palabras bajo cláusulas de intangibilidad, las cuales son absolutas e insalvables frente a la actuación del poder de revisión de constitucional, en este orden de ideas el autor también hace referencia a los límites materiales implícitos, los cuales radican en las decisiones políticas fundamentales del soberano y a los límites formales que se encuentran plasmados en el propio procedimiento establecido para la modificación constitucional.

El autor finaliza este apartado sosteniendo que en un Estado Constitucional democrático y social es el Pueblo y sólo el Pueblo quien es el único legitimado para decidir sobre la forma y modo de su gobierno, acto que se realiza a través del Poder Constituyente y que asegura mediante el establecimiento de medidas jurídicas que ha de adoptar la Comunidad Política. El propio autor señala que Pedro De Vega y él han sostenido la idea de que el Pueblo como ente soberano no puede ser separado de la toma de decisiones políticas fundamentales una vez que la Constitución haya entrado en vigor.

Indiscutiblemente la magnífica obra que brevemente se ha expuesto constituye un estudio muy bien detallado, coherente y preciso sobre la Reforma Constitucional, la cual es ampliamente recomendable para que sea estudiada por los profesionales científicos del Derecho, no únicamente constitucionalistas si no también cualquier otro jurista, en virtud de que la Reforma Constitucional impacta en todo el orden jurídico de un Estado. Esta obra es de estudio obligatorio para aquellos investigadores de toda Iberoamérica, en virtud de que sienta las bases metodológicas para comprender ese mecanismo constitucional e incluso para llevarlo al ámbito práctico. 